\title{
The Role of Self-Produced Movement and Visual Tracking in Infant Spatial Orientation
}

\author{
Linda P. Acredolo, Anne Adams, and Susan W. Goodwyn \\ University of California, Davis
}

\begin{abstract}
In two longitudinal studies, infants were trained at 12 and 18 months to find an object hidden in one of two identical wells in a Plexiglas box. On the test trial, normal access was blocked and infants were either guided by their mother or allowed to move on their own to another opening on the opposite side. In Experiment 1 significantly more correct responding occurred after active movement than after passive at 12 months, with correct responding related to high visual tracking. In contrast, at 18 months correct search without tracking predominated among both movement conditions. A difference between the conditions in the position of the mother on the test trial was ruled out as a contributor to performance on the basis of data from Experiment 2. When opaque sides were inserted to prevent tracking in Experiment 3, active movement no longer facilitated correct search at 12 months, thus indicating that the tracking and not the active movement per se was the critical factor.
\end{abstract}

To date an impressive body of research has accumulated indicating that the younger the infant the more likely he or she is to give priority to egocentric over objective spatial information when trying to remember locations in large-scale spatial environments. In four separate studies, Acredolo (1978), Acredolo and Evans (1980), Cornell and Heth (1979), and Rieser (1979) each noted strong tendencies by infants 6 months or younger to look, in anticipation of an event, in the same direction as had been appropriate before the infant had been moved to a different spot in the environment. Acredolo (1978) and Cornell and Heth (1979) also included older age groups and, consequently, were able to demonstrate that this tendency declines as age increases.

Although the exact nature of this "egocentric" behavior has been debated, that is, whether it reflects dependence on a body-centered organization of space or simply a motor habit (see Acredolo, in press, for a review), the fact remains that infants are increasingly willing or able as they grow older to forego reliance on such information in favor of

Requests for reprints should be sent to Dr. Linda P. Acredolo, Department of Psychology, University of California, Davis, CA 95616. 
reliance on landmarks and/or truly coordinated perspectives of the spaces around them. One obvious question that remains is "Why?" What is it that enables or motivates the older infant but not the younger to look beyond the relation of objects and events to his or her own body? It is our belief that a parallel between development in this domain and the development of locomotor skills may provide some clues to this developmental mystery.

Recognition of parallel development in these two domains is not new. As early as 1977 Bremner and Bryant pointed out that the rise in objective responding in spatial tasks of this sort cooccurs with changes from sitting to crawling to walking-an observation echoed by Acredolo in 1978 . Speculation about a causal relation between the two is tantalizingly plausible, but without some direct evidence of a link between self-produced movement and objective responding, must remain just that-speculation. It was the purpose of the present experiment to provide such evidence, and to do so through the use of a longitudinal design which would allow comparison of early and late locomotor periods.

Our faith in the idea of a link between locomotor development and objective responding is strengthened by existing research with infants showing relationships between self-produced movement and other spatial phenomena. For example, Campos, Svejda, Bertenthal, Benson, and Schmid (1981) have demonstrated that at least some experience with selfproduced movement, either in the form of crawling or experience in a "walker" (i.e., an apparatus on wheels which allows even a noncrawling infant to move through space) is necessary for development of the degree of depth perception exhibited by infants who avoid the deep side of a visual cliff. Apparently, moving themselves through space accomplishes for this aspect of spatial knowledge something that passive movement (being carried from place to place) does not. Exactly what that contribution is, is not yet totally clear, although an increase in visual attention to the environment is hypothesized by Campos et al. (1981) to be an important factor.

If visual attention is indeed an important product of self-produced movement, the question remains: Attention to what? At least a partial answer may be attention to the different visual perspectives that result from movement. This suggestion is supported by another study by Campos (Campos, Bertenthal, \& Benson, 1980) in which crawling and noncrawling $7 \frac{1}{2}$-month-old infants were compared on their ability to extract a constant form from a fluctuating display. Using a habituation paradigm similar to one used by Ruff (1978), Campos et al. (1980) demonstrated that the infants with crawling experience were more likely than noncrawling infants to recognize a form as familiar even though it had been presented from a variety of different viewpoints. If Campos et al. (1980) are indeed correct in interpreting this pattern as indicative of a link between selfproduced movement and a tendency to coordinate perspectives, then it 
seems reasonable to expect self-produced movement to have an equally facilitative effect on the type of spatial orientation task at issue here. After all, an objective response in studies like those of Acredolo (1978) is really a recognition by the infant of "place constancy," analogous to the "shape constancy" studied by Campos et al. (1980).

One final study suggesting we are correct in hypothesizing a connection between self-produced movement and spatial orientation in infants is a brief report of a creative study by Benson and Uzgiris (1981). Elevenmonth-old infants were taught to find an object in one of two hiding places in a large, three-sided Plexiglas box. During the training trials the permanently open side was the side farthest from the infants and the wall closest to the infants was removed to allow access to the two covered wells. During the test trials which followed training, the object was once again hidden in the same well, the wall closest to the infants was replaced, and the infants were either carried or allowed to move on their own to the opposite, permanently open side of the box in order to retrieve the object. Just as we would predict, the results indicated significantly fewer egocentric choices on the test trials after "active" movement.

Several factors, however, make us cautious about accepting these results as definitive. It appears that the active and passive conditions differed in several important respects in addition to the type of movement, differences which may have favored the active condition. Specifically, in the active condition infants remained at ground level and could reach for the object as soon as the far corner of the box was rounded. Thus, visual tracking of the correct site was fairly easy to maintain, and the infants were never again confronted with the confusing "one-on-theright, one-on-the-left" configuration that they would have seen had they been required to reach the middle of the open side before searching. In contrast, in the passive condition, infants were apparently lifted off the ground and set down in the middle of the open side. Consequently, their view of the apparatus was disrupted and their next ground level view was in an important way a replication of their original view: one hiding spot to their right, one to their left. It is conceivable that such a combination could have increased the likelihood of dependence on egocentric information. These differences between the active and passive conditions were eliminated in the present study, and a longitudinal design assessing performance at both 12 and 18 months was used. The hypothesis, however, remained the same-that active, self-produced movement would facilitate the ability of infants to coordinate perspectives and respond objectively.

\section{EXPERIMENT 1}

\section{Method}

Subjects. Subjects were twenty-three, $12 \frac{1}{2}$-month-old infants, twelve boys and eleven girls, ranging in age at first testing from 12.4 to 13 
months with a mean of 12.6 months $(S D=4.6$ days $)$. Their names were drawn from birth announcements in papers from predominantly middleclass communities in northern California. All twenty-three of these infants returned 6 months later for retesting. Their ages at that time ranged from 18.4 to 19.3 months with a mean of 18.8 months ( $S D=8.6$ days).

Materials. The major piece of equipment used in the study was a 0.96 $\times 0.63-\mathrm{m}$ box $(38 \times 25$ in.), $0.63 \mathrm{~m}$ high, made of wood and Plexiglas (see Fig. 1). The wooden base of the box was $12.7 \mathrm{~cm}(5 \mathrm{in}$.) off the ground so that it could contain two $12.7 \times 12.7-\mathrm{cm}$ hiding wells, 30.5 $\mathrm{cm}$ (12 in.) apart, from center to center. The four corners of the box consisted of wooden tracks into which Plexiglas walls were inserted. In all conditions of the study one of the two long walls (called the front) was broken in the middle by a $30.5-\mathrm{cm}$ (12 in.) opening through which the children could reach the hiding wells. The placement of the opening in the middle of the front wall ensured that the subjects would be viewing the wells with one to their left and the other to their right as they reached in to retrieve the object. The toys used in the hiding portion of the study included a Donald Duck top and a wind-up train. The only other materials were two white cloth diapers used to cover the hiding wells, and a round fence called a corral, $3.04 \mathrm{~m}(10 \mathrm{ft})$ in diameter, used to keep the children from wandering away from the apparatus.

Procedure. Infants were randomly assigned to one of the two groups with the result that 11 infants were tested in the active condition ( 5 boys and 6 girls) and 12 in the passive condition ( 7 boys and 5 girls). Their condition assignments remained the same at the 18 month testing session. At 12 months, 8 infants in each condition were walkers. Average age when walking began was 10.4 months for the active infants and 10.7 for the passive; these means were not significantly different.

The same experimental procedure was used at both ages. After an initial familiarization period inside the corral, the detour nature of the

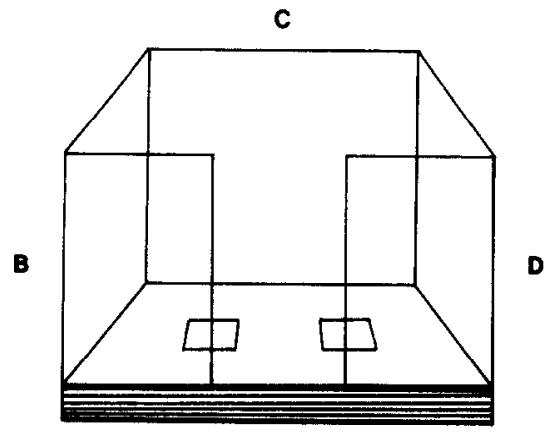

A

FIg. 1. Experimental box: Wall $\mathrm{A}=$ front, Wall $\mathrm{C}=$ back. 
box was demonstrated to each subject. A toy was placed inside the box so that it was still visible from all angles and the child was placed just around the corner of the box. After the toy was retrieved, the same procedure was repeated, this time with the child closer to the back of the box. Finally, the child was placed in the middle of the back of the box, directly opposite the opening, and required to retrieve the toy three times by crawling or walking around the box to the opening. This movement was analogous to what would be required of subjects in the active condition and ensured that all subjects understood that the box was constructed to always allow access from one side. The direction in which the child was trained to go around the box was varied systematically across children. The hiding phase of the experiment was then begun.

At least five training trials and one test trial constituted the main phase of the experiment. Throughout all but the very end of this period the infant was seated in the middle of the long, back side of the box directly opposite the opening. His or her parent was seated behind the child, with the experimenter to the child's left or right. Access to the well for the training trials was provided by removing the Plexiglas wall.

The procedure for each of the training trials was the same. As the child was held back by the parent, the experimenter hid a toy in one of the two cloth-covered wells. The right well was used for half the infants in each condition, the left for the other half. The hiding place chosen was always on the side of the box around which the child had been trained to go during the familiarization phase. The object was hidden in the same well and the infant was encouraged to retrieve the toy and play with it until the child had successfully retrieved the toy five times. The test trial followed immediately.

The test trial was initiated by the replacement of the Plexiglas wall, thus denying the subjects direct access to the wells. The experimenter then moved around to the opening and hid the toy in the same well used for the training trials while the infant watched from the back of the box. Once the object was in place, one of two things happened. Infants in the active condition were set on their feet by their parent and encouraged to find the object. As the child moved around the box, the parent remained in her original place and the experimenter remained in the vicinity of the opening, far enough back to allow the child easy access. Infants in the passive condition were set on their feet by their parents, held under their arms, and carried around the box to the opening with their feet close to the floor. Parents were told that the child's path should be the same as though he or she were walking. As the child searched for the object from the new position, the parent remained directly behind the child and the experimenter moved to the parent's original position at the back of the box. Consequently, subjects in both conditions experienced 
one adult across from them and one adult behind them while they searched on the test trial.

Two types of data were collected on the test trial. The first was an evaluation of the infant's visual behavior as they moved around the box. Both the experimenter and another observer rated the degree to which the infants tracked the toy's location using the following scale: $0=$ no tracking at all; $1=$ occasional glances into the box; $2=$ occasional glances away from the box; 3 = constant tracking throughout movement. The second measure was the accuracy of the child's search. The observer noted whether or not the infant succeeded in finding the toy (objective response) or instead chose the hiding place consistent with reliance on an egocentric frame of reference (egocentric response).

\section{Results and Discussion}

Relevant data for each subject at each age included the number of training trials to criterion, the nature of the child's choice on the test trial, and the degree to which the child visually tracked the hidden object during movement to the other side of the box. Each point will be addressed in turn.

Training trials continued until each subject had successfully retrieved the toy five times. Overall, $92 \%$ of the subjects reached this criterion in five trials at 12 months and $95 \%$ at 18 months. The remaining infants took six or seven trials and were evenly distributed across conditions.

Responses on the test trial were categorized as egocentric (incorrect) or objective (correct). Presented in Table 1 are the numbers of children in the active and passive conditions falling into four response patterns, each pattern representative of a possible combination of responses at the two ages tested. For example, the EE category includes those subjects who responded egocentrically at both ages, and the EO category those who responded egocentrically at 12 months but objectively at 18 months.

Assuming that chance alone was operating, one would expect an equal number of subjects to fall into each category. However, a binomial test based on a probability of .25 that a subject would fall into a given

TABLE 1

Number of Children in Experiment 1 Exhibiting Each of Four Possible Response Patterns on Test Trials across Two Ages

\begin{tabular}{lccccc}
\hline & \multicolumn{5}{c}{ Response pattern } \\
\cline { 2 - 5 } Condition & EE & EO & OE & OO & Total \\
\hline Active & 1 & 2 & 1 & 7 & 11 \\
Passive & 1 & 8 & 1 & 2 & 12 \\
\hline
\end{tabular}

Note. $\mathrm{E}=$ egocentric response; $\mathrm{O}=$ objective response; first letter represents response at 12 months; second letter represents response at 18 months. 
category, indicated that the number of subjects in the active condition falling into the $\mathrm{OO}$ category was significantly greater than chance alone would predict $(p=.008)$. In other words, a significant proportion of subjects in the active condition were able to find the object on the test trial at both ages. For the passive condition, significantly more subjects than would be expected by chance fell into the EO category $(p=.003)$. Thus, in contrast to the subjects in the active condition, those in the passive condition tended to respond egocentrically at 12 months and then shift to objective responding at 18 months. A McNemar test of related samples also supported this finding of a developmental shift among subjects in the passive condition $\chi^{2}(1, N=12)=4.00, p<.05$. In addition, the difference between the active and passive conditions was demonstrated directly in a comparison of performance at 12 months: a Fisher's exact test indicated a significantly greater proportion of objective responders among subjects in the active than among subjects in the passive condition $(p<.05)$.

In sum, the results from the 12-month-olds of Experiment 1 support the hypothesis that active, self-produced movement facilitates objective responding in a simple spatial task requiring coordination of two perspectives. However, this facilitative effect no longer operates in this task at 18 months due to a ceiling effect; at this age the task was easily solved after either active or passive movement.

The question of what role visual tracking played in these results remains to be answered. The visual tracking scores were based on a 4-point rating scale (0-3) for which interrater reliability was $95 \%$. The few discrepancies between observers were resolved through discussion. For purposes of analysis the values of the scale were collapsed into two categories: 0 and 1 = high tracking; 2 and 3 = low tracking. Presented in Table 2

TABLE 2

Number of Subjects at Each Age of Experiment 1 Exhibiting Each of Four Possible Combinations of Visual Tracking Behayior and Search

\begin{tabular}{cccc}
\hline & & \multicolumn{2}{c}{ Response } \\
\cline { 3 - 4 } & Tracking & Egocentric & Objective \\
\hline 12 months & Low & 3 & \\
Active & High & 0 & 1 \\
& Low & 9 & 7 \\
Passive & High & 0 & 2 \\
& & & 1 \\
18 months & Low & 2 & 8 \\
Active & High & 0 & 1 \\
Passive & Low & 2 & 9 \\
& High & 0 & 1
\end{tabular}


are the numbers of children at each age exhibiting each of four possible combinations of visual tracking (low versus high) and search (egocentric versus objective). Statistical analyses (i.e., Fisher's exact tests and binomial tests) applied to the frequency data in Table 2 reveal a number of patterns significant at $p<.05$ or better. First, the data indicate that at 12 months, subjects in the active condition were more likely to track efficiently than those in the passive condition. Moreover, this tracking behavior seems to have been helpful to the infants. Across both the active and passive conditions and within the active condition alone, those infants who successfully found the object on the test trial (i.e., responded objectively) were significantly more likely to show high tracking than low tracking. ${ }^{1}$ The reverse was also true at 12 months: those infants, both across conditions and within the passive condition, who responded egocentrically (i.e., did not find the object) were significantly more likely to show low levels of tracking than high. Thus, the data at 12 months are consistent with the hypothesis that visual tracking is more likely to occur with active than with passive movement. Moreover, high levels of tracking are correlated with correct, objective search at this age.

Table 2 also reveals, however, that these relations among movement, search, and tracking do not continue at 18 months. Two things appear to have happened to change them. The first, a shift toward objective responding among the passive group, has already been discussed. The second is a shift toward low levels of tracking in the active condition where tracking was originally high. In support of this conclusion, binomial tests indicated that the proportion of active subject at 18 months showing low levels of tracking was significantly greater than would be expected by chance, both across response categories $(p<.005)$ and within the subgroups responding objectively $(p$ 's $<.05)$. When one puts these two changes together, along with the continuation of objective responding among the active subjects and low tracking among the passive subjects, the following picture emerges: the children at 18 months were easily able to keep track of the location of the hidden object no matter what kind of movement was involved. Moreover, few if any infants bothered to track the location of the object as they moved.

The validity of this description of developmental change is also supported by the data as displayed in Table 3 . Unlike Table 2 , the data in Table 3 are grouped so as to show patterns of change across age in tracking

\footnotetext{
${ }^{1}$ An interesting exception to this pattern is the fact that two of the three infants in the passive condition who searched correctly did so despite low levels of tracking. Unfortunately it is impossible with such small number to tell whether the behavior of these subjects was due to chance alone or to superior spatial skill. At this point there is no obvious reason to suspect the latter. In fact, one of these two subjects was among the few subjects who did not search correctly at 18 months. Superior ability seems particularly unlikely in this case.
} 
TABLE 3

Number of Children in Experiment 1 Falling into Sixteen Possible Combinations of Visual Tracking and Response Patterns

\begin{tabular}{lcccccr}
\hline \multirow{2}{*}{ Condition } & Tracking & \multicolumn{5}{c}{ Response patterns } \\
\cline { 3 - 7 } Active & pattern & EE & EO & OE & OO & Total \\
& Lo/Lo & 1 & 2 & 0 & 1 & 4 \\
& Lo/Hi & 0 & 0 & 0 & 0 & 0 \\
\multirow{2}{*}{ Passive } & Hi/Lo & 0 & 0 & 1 & 5 & 6 \\
& Hi/Hi & 0 & 0 & 0 & 1 & 1 \\
& Lo/Lo & 1 & 8 & 1 & 1 & 11 \\
& Lo/Hi & 0 & 0 & 0 & 0 & 0 \\
& Hi/Lo & 0 & 0 & 0 & 1 & 1 \\
& Hi/Hi & 0 & 0 & 0 & 0 & 0 \\
\hline
\end{tabular}

Note. $\mathrm{E}=$ egocentric response; $\mathrm{O}$ - objective response. First entry in pattern label indicates behavior at 12 months; second entry indicates behavior at 18 months.

and search for each individual subject. For example, the Hi/Lo tracking pattern includes those subjects who showed a high degree of visual tracking at 12 months but a low degree at 18 months, and the EE pattern includes subjects who responded egocentrically at both ages. Grouped in this way, the data are particularly clear in showing that visual tracking in the active condition declines with age: the $\mathrm{Hi} / \mathrm{Lo}$ pattern is significantly more prevalent than would be expected by chance $(p<.05)$.

\section{EXPERIMENT 2}

The results of Experiment 1 demonstrated that objective responding was more likely among infants in the active condition than the passive. However, in addition to the type of movement, these two conditions also differed in terms of the mother's position on the test trial. Mothers of infants in the active condition remained in their original position on Side $\mathrm{C}$, whereas mothers of infants in the passive condition moved with their infants to Side A. The purpose of Experiment 2 was to determine whether the difference in search behavior between the active and passive groups of Experiment 1 could be explained on the basis of this difference rather than the type of movement involved.

\section{Method}

Subjects included six 12-month-old infants (four males and two females) who had not been tested in Experiment 1 . The apparatus and procedures used were identical to those of Experiment 1 with the exception that the infants were allowed to move themselves around the box on their own on the test trial while their mothers followed. Thus, all six infants were 
tested in the active condition with the mothers' position the same as experienced by infants in the passive condition of Experiment $1 .^{2}$

\section{Results and Discussion}

Five out of the six infants reached the training criterion of five successful searches in five trials. The 6 th infant needed six trials. On the test trial, 5 out of the 6 infants searched accurately (objectively). A Fisher exact probability test was used to compare these data with those of the passive condition of Experiment 1 in which only 3 of 12 infants had searched accurately. The results indicated a significantly higher proportion of correct responses among the active subjects of Experiment 2 than among the passive subjects of Experiment $1(p=.04)$. Thus, the type of movement and not the mother's position on the test trial appears to be the determining factor. These results do not support a suggestion made by Presson and Ihrig (1983) that egocentric search in paradigms of this sort is due to the child's reliance on the mother's maintenance of her position relative to the child as a cue that his or her own position in space has not changed and that the original search response is still appropriate.

\section{EXPERIMENT 3}

The results of Experiment 1 also demonstrated that visual tracking is more likely to occur with active, self-produced movement at 12 months than with passive, other-directed movement. Moreover, both visual tracking and active movement are associated with objective search behavior at this age. It is very tempting to conclude from this pattern that it is the visual tracking at 12 months which facilitates objective responding, and that the advantage of active movement over passive at this age is due to active movement, for some as yet undetermined reason, tending to promote visual tracking while passive movement does not. Indeed, it was just this conclusion to which Campos, Svejda, Campos, and Bertenthal (1982) came based on their observations of locomotor and nonlocomotor infants: "it has been our impression that the infant who is passively carried is in a state of 'visual idle'-staring blankly straight ahead and not focusing on single objects in the environment" (p. 208).

\footnotetext{
${ }^{2}$ Another logical possibility would have been to run the six subjects in a passive condition with the mother maintaining her original position. Such a group could then have been compared to the active group from Experiment 1 who also experienced a stationary mother. Unfortunately, allowing the mother to maintain her position in the passive condition would have made it necessary for someone else to move the infant to the other side of the box. It seemed likely that the sudden intrusion of a stranger in this way could create levels of anxiety high enough to prevent adequate attention to the task. Any egocentric responses that resulted could be due to this anxiety rather than to the passive movement per se. Because of this potential confound, we decided to run the six subjects in an altered version of the active rather than the passive condition.
} 
However convincing such impressions might be, they unfortunately do not constitute proof that it is the tracking, and not the active movement per se, that most directly facilitates objective responding in our task. After all, it is entirely possible that the visual tracking is coincidental and that it is really the motor feedback from the self-produced movement which enables the 12-month-old infants in the active condition to maintain their orientation to the task materials. Indeed, the fact that at 18 months objective responding predominated despite little or no tracking behavior, could be interpreted as evidence supportive of this latter hypothesis.

It was the purpose of Experiment 3 to explore more precisely the relation among active movement, visual tracking, and objective responding. To this end, the transparent side walls of the box were replaced with opaque walls in order to disrupt the infants' attempts to keep their eyes on the target as they moved themselves around the box. If the infants, despite this disruption, were still able to find the object, then the results would suggest that active movement per se and not visual tracking is the crucial element. However, should the number of subjects searching correctly significantly decline in comparison to the active condition of Experiment 1, then it would seem likely that visual tracking and not active movement is the more direct facilitator of objective responding in this situation.

\section{Method}

The subjects included 13 infants ( 7 boys and 6 girls), tested first at 12 months and later at $\mathbf{1 8}$ months of age. Their ages at the initial test ranged from 12.4 to 13.1 months with a mean of 12.7 months ( $S D=5.98$ days). At the second test the age range was 18.4 to 19.0 months with a mean of 18.69 months ( $S D=4.8$ days). None of the infants in Experiment 3 had been included in Experiment 1 or 2. Twelve of the infants were walkers; average age at onset of walking was 10.6 months.

The materials were the same as those used in Experiment 1 with the exception that the Plexiglas walls on the two short sides of the box were replaced with fiberboard. The procedure used at both testing sessions was identical to that used in Experiment 1 with the exception that the infants were only tested in the active, not the passive, condition. The mother remained in her original position on Side $C$, and the experimenter in the vicinity of the opening.

\section{Results}

The criterion for training was five successful searches from the initial position. All but two of the infants reached this criterion in five trials; the remaining two took six trials. Once again the infants' choices on the test trial were categorized as objective (i.e., correct) or egocentric (i.e., incorrect), and the results for each subject for the two ages were combined 
into four patterns. Of the 13 subjects, 1 exhibited the EE pattern, 8 exhibited the EO pattern, 1 exhibited the OE pattern, and 3 exhibited the $O O$ pattern. A binomial test applied to these data indicated that more subjects than would be expected by chance fell into the EO category ( $p$ $<.01$ ), the pattern indicative of a shift from egocentric responding at 12 months to objective responding at 18 months. A McNemar's test of related samples also indicated that this shift was statisically significant, $\chi^{2}(1, N=13), p<.05$.

Fisher's exact probability tests were used to compare the data from the opaque condition of Experiment 2 with the data from the active and passive transparent conditions of Experiment 1. These analyses revealed that the proportions of egocentric and objective responders in Experiment 2 did not differ significantly from the proportions among the passive subjects of Experiment 1 at either age. However, there was a significant difference between the two active groups at 12 months, the opaque condition of Experiment 2 producing a higher proportion of egocentric responders than the transparent condition of Experiment $1(p<.05)$. The shift to objective responding at 18 months by the infants in the opaque condition eliminated this difference.

The pattern which emerges from a comparison of the two experiments suggests quite strongly that active movement per se is not enough to guarantee objective responding at 12 months, at least not in our task. Twelve-month-old infants who were allowed to move on their own to the other side of the box, but whose efforts to track the location of the object were thwarted by opaque walls, were no better at finding the toy than the 12-month-old passive subjects of Experiment 1. It seems clear, then, that the visual tracking behavior exhibited at 12 months by the active subjects of Experiment 1 was a very important contributor to the high proportion of objective responders that resulted.

\section{GENERAL DISCUSSION}

The purpose of the present studies was to investigate the role active, self-produced movement might play in the type of rotation task typically used to assess spatial orientation during infancy. The results indicated that, at least at 12 months, spatial orientation was indeed faciliated by allowing the infants to move through the space on their own. The subjects in the active condition were significantly more likely than those in the passive condition to find the object on the test trial. These results for the 12-month-olds are clearly in line with the conclusions drawn by Benson and Uzgiris (1981) and Campos et al., (1982) based on their own investigations of the contribution that self-produced movement makes to infant spatial behavior. They also extend to infancy the pattern that has consistently emerged from studies of older children and adults (e.g., Acredolo \& Feldman, 1979; Appleyard, 1970; Cohen, 1982). It appears 
that the facilitating effects of self-produced movement represent a continuity across development.

The present results, however, allow us to go beyond merely cataloging the existence of a relationship between active movement and spatial orientation during infancy. They also provide important insights into why the relationship exists. By recording the tracking behavior of the infants as they moved themselves or were moved around the box by their mothers, we were able to detect a highly significant three-way relationship at 12 months among objective responding, active movement, and visual tracking: those infants who tracked were more likely to find the object; those infants who moved themselves were more likely to find the object; and those infants who moved themselves were more likely to track. Moreover, the results of Experiment 3, in which the infants' view into the box was blocked, provided evidence that it was the tracking and not the movement itself that most directly facilitated objective responding at 12 months. Self-produced movement appears to aid the infant by increasing attention to relevant environmental information.

Of course, it is possible that our emphasis should not be on the possibility that active movement promotes tracking, but rather on the equally plausible hypothesis that being carried by an adult hinders tracking. This is a subtle but potentially important distinction. The implication for the present study is that the active/passive effect obtained may be very specific to passive movement involving being picked up by an adult. It may be that infants come to feel they have no control over where they go next in such situations, and that therefore no purpose is served by maintaining orientation to where they have just been. It is possible that visual tracking would be maintained under other conditions of passive movement, for example, when such movement still allows infants to predict their next location in space. Unfortunately, at this point all we can do is point out the distinction in the hope that others will provide data to clarify the issue.

The existence of these alternative explanations for our results does not diminish the importance of the discovery that tracking is related to superior performance on a spatial task at 12 months. In this sense our results are consistent with the results of several studies with infants. Both Goldfield and Dickerson (1981) and Bremner (1978) found that 9month-old infants who visually tracked a target location as they were rotated around a table were more likely to search correctly (i.e., objectively). Visual tracking among the infants in the former study, however, was not very frequent when the two containers on the table were identical. Many of the infants at this age seemed to need a distinctive landmark to "hang on to" as they were moved. Bremner's data hint at another factor which may facilitate tracking in orientation tasks of this type. Successful search by his infants was correlated with both tracking and 
the elimination of training trials. It seems quite likely that the elimination of the training trials increased tracking. Why might that be? Acredolo (in press) suggests it may simply be that young infants find it easier to rely on well-practiced responses, such as result from training trials, than to make the effort to visually track.

The picture emerging from these two studies in combination with our own is that visual tracking is a crucial contributor to accurate spatial behavior from 9 to at least 13 months. Effective tracking, however, is not automatic. It tends to occur more often with active than with passive movement, with differentiated than with undifferentiated displays, and with less rather than with more experience searching in a particular direction relative to the body. Moreover, it seems unlikely that this list is exhaustive. It could well be, for example, that the infant's motivation to find an object would affect visual tracking. A highly valued object might induce a high degree of tracking even under conditions of passive movement, while a less valued object would not. It is also quite likely that the infant's anxiety level will affect tracking behavior. An infant who is frightened or ill at ease will not be as well able to organize effective attentional strategies. In fact, the results of two studies comparing the effect familiar versus unfamiliar environments have on performance in this type of task (Acredolo, 1979, 1982), provide indirect evidence to support such an hypothesis.

To this point we have focused exclusively on the results from the 12month testing session, using the pattern we observed as evidence that visual tracking plays a role in infant spatial behavior. Of equal importance to the emerging storyline, however, is the fact that the relationships seen so clearly at 12 months disappeared completely at 18 months, being replaced by almost universal objective responding without the benefit of visual tracking. How can we account for such a dramatic shift? It seems quite likely that the development of mental representation skills (i.e., the symbolic function) plays a large role. In contrast to the 12-montholds who had to keep their eyes on the target as they changed perspectives, at 18 months the infants were able to mentally represent the simple spatial relations involved in our task and easily predict the consequences of their movement. Such behavior is very reminiscent of some of Piaget's own descriptions of Stage 6 spatial behavior (Piaget, 1954). At 18 months, for example, his daughter Lucienne executed a complicated detour around a room in order to reach him without having to let go of the furniture she felt she needed to support her walking. Piaget's observation that, "The whole journey is made without looking at me" (p. 232), is clearly analogous to our own observations of infant spatial behavior at 18 months. At least under the simple conditions represented by our paradigm, "the whole journey" can be made at this age without even looking at the box. The infants have moved from reliance on direct perception to internal 
representations which allow the coordination of simple perspectives. It is our belief that this achievement is due at least in part to the mountains of information about spatial relations that visual tracking had provided over the previous months.

Of course, just because our 18-month-olds did not need to rely on tracking to solve our task does not mean that they would not need to do so in order to solve a task involving more complex spatial relations. What we suggest may remain constant across age and complexity is the ordering we observed so clearly within our simple task: purposeful attentional strategies (especially under conditions of active movement) followed by more automatic, internal, spatial problem solving. In fact, this was exactly the pattern found by Poag, Cohen, and Weatherford (1983) in their study of 5- and 7-year-old children required to remember the spatial layout of an entire room. It was only the 5-year-olds for whom self- versus other-directed movement and self- versus other-directed viewing made a difference. At this age, self-directed movement through the room resulted in superior recall regardless of whether the experimenter directed attention to salient features or not. Who controlled visual attention did, however, make a difference for 5-year-olds who were led through the environment by an adult. Among these subjects, self-directed viewing led to less accurate spatial memory than adult-directed viewing. In contrast, the 7-year-old subjects performed comparably across all conditions. This pattern of developmental change is entirely consistent with our own. At the younger age, active movement facilitated spatial knowledge; adult direction of attention was not needed because the children were already looking in the right places. In contrast, other-directed movement, like the passive condition of our own study, resulted in poor performance when the younger children's visual behavior was left entirely in their own hands. Only when an adult stepped in and specifically directed their attention as well as their movement, did these "passive" subjects retain adequate knowledge of the space. The older subjects, like our 18-montholds, showed no such limitations. Thus, just as in every other domain of intellectual development, the nature of the task will in part determine the specific age differences one can expect.

\section{REFERENCES}

Acredolo, L. P. (1978). Development of spatial orientation in infancy. Developmental Psychology, 14, 224-234.

Acredolo, L. P. (1979). Laboratory versus home: The effect of environment on the 9month-old infant's choice of spatial reference system. Developmental Psychology, 15, 666-667.

Acredolo, L. P. (1982). The familiarity factor in spatial research: What does it breed besides contempt? In R. Cohen (Ed.), Children's perceptions of spatial relations. (pp. 19-30). San Francisco: Jossey-Bass.

Acredolo, L. P. (in press). Coordinating perspectives on infant spatial orientation. In $\mathbf{R}$. Cohen (Ed.), The development of spatial cognition. New York: Erlbaum. 
Acredolo, L. P., \& Evans, D. (1980). Developmental changes in the effects of landmarks on infant spatial behavior. Developmental Psychology, 16, 312-318.

Appleyard, D. A. (1970). Styles and methods of structuring a city. Environment and Behavior, 2, 100-116.

Benson, J. B., \& Uzgiris, I. C. (1981). The role of self-produced movement in spatial understanding. Paper presented at the biennial meetings of the Society for Research in Child Development, Boston, MA.

Bremner, J. G. (1978). Egocentric versus allocentric spatial coding in 9-month-old infants: Factors influencing the choice of code. Developmental Psychology, 14, 346-355.

Bremner, J. G. \& Bryant, P. E. (1977). Place versus response as the basis of spatial errors made by young infants. Journal of Experimental Child Psychology, 23, 162-171.

Campos, J., Bertenthal, B., \& Benson, N. (1980). Self-produced locomotion and the extraction of form invariance. Paper presented at the meetings of International Conference on Infant Studies, New Haven, CT.

Campos, J., Svejda, M., Bertenthal, B., Benson, N., \& Schmid, D. (1981). Self-produced locomotion and wariness of heights: New evidence from training studies. Paper presented at the meetings of the Society for Research in Child Development, Boston, MA.

Campos, J. J., Svejda, M. J., Campos, R. G., \& Bertenthal, B. (1982). The emergence of self-produced locomotion: Its importance for psychological development in infancy. In D. Bricker (Ed.), Intervention with at-risk and handicapped infants. Baltimore, MD: Univ. Park Press.

Cohen, R. (1982). The role of activity in the construction of spatial representations. In $\mathbf{R}$. Cohen (Ed.), Children's conceptions of spatial relations. (pp. 41-55). San Francisco: Jossey-Bass.

Cornell, E. H., \& Heth, C. D. (1979). Response versus place learning by human infants. Journal of Experimental Psychology: Human Learning and Performance. 5, 188-196.

Feldman, A., \& Acredolo, L. P. (1979). The effect of active versus passive exploration on memory for spatial location in children. Child Development, 50, 698-704.

Goldfield, E. C., \& Dickerson, D. J. (1981). Keeping track of locations during movement of 8- to 10-month-old infants. Journal of Experimental Child Psychology, 32, 48-64.

Piaget, J. (1954). The construction of reality in the child. New York: Basic Books.

Poag, C. K., Cohen, R., \& Weatherford, D. L. (1983). Spatial representations of young children: The role of self- versus adult-directed movement and viewing. Journal of Experimental Child Psychology, 35, 172-179.

Presson, C. C., \& Ihrig, L. H. (1983). Using mother as a spatial landmark: Evidence against egocentric coding in infancy. Developmental Psychology, 18, 699-704.

Rieser, J. (1979). Spatial orientation of six-month-old infants. Child Development, 50, 10781087.

Ruff, H. (1978). Infants' recognition of the invariant forms of objects. Child Development, 49, 293-306.

ReCeIved: July 25, 1983; REvised: January 31, 1984. 